\title{
Thirty-day mortality after hip fractures: has anything changed?
}

\author{
Dionysios Giannoulis ${ }^{1}$ - Giorgio M. Calori ${ }^{2}$ - Peter V. Giannoudis ${ }^{1,3}$
}

Received: 17 January 2016/Accepted: 21 January 2016/Published online: 4 March 2016

(C) The Author(s) 2016. This article is published with open access at Springerlink.com

\begin{abstract}
Bone density insufficiency is the main cause for significant musculoskeletal trauma in the elderly population following low-energy falls. Hip fractures, in particular, represent an important public health concern taking into account the complicated needs of the patients due to their medical comorbidities as well as their rehabilitation and social demands. The annual cost for the care of these patients is estimated at around 2 billion pounds $(\mathfrak{E})$ in the UK and is ever growing. An increased early and late mortality rate is also recognised in these injuries together with significant adversities for the patients. Lately, in order to improve the outcomes of this special cohort of patients, fast-track care pathways and government initiatives have been implemented. It appears that these measures have contributed in a steady year-by-year reduction of the 30-day mortality rates. Whether we have currently reached a plateau or whether an ongoing reduction in mortality rates will continue to be observed is yet to be seen.
\end{abstract}

Keywords Hip fracture - Complications - Mortality · Early surgery $\cdot$ Fast-track pathways

Peter V. Giannoudis

pgiannoudi@aol.com

1 Academic Department of Trauma and Orthopaedics Surgery, School of Medicine, University of Leeds, Leeds, UK

2 Academic Department of Trauma and Orthopaedics, School of Medicine, University of Milan, Milan, Italy

3 NIHR Leeds Biomedical Research Unit, Chapel Allerton Hospital, Leeds, West Yorkshire LS7 4SA, UK

\section{Introduction}

Hip fractures continue to be a significant injury in the elderly population [1]. They represent an important public health concern taking into account the complicated needs of the patients due to their medical comorbidities as well as their rehabilitation and social demands [2].

Data from studies that report a rise in the number of the elderly in the future predict also an increase in the number of hip injuries that will require treatment [3]. In the USA, elderly are the fastest growing category of the population with people above the age of 65 years or age expected to reach an estimated value of 89 million by year 2050 [4]. In the UK, by 2020 there is an estimate of 101,000 annual cases of patients with hip fractures [5]. The majority of the patients with hip fractures are women (approximately $75 \%$ ) with an average age of 80 years [6].

Hip fractures are associated with increased morbidity and significant financial cost implications [7]. The annual cost for the care of these patients in the UK is estimated at around 2 billion pounds ( $($ ) and is ever growing [8]. In the USA, approximately $\$ 40,000$ are spent in the first year for every patient with hip fracture for direct medical costs [9]. An increased early and late mortality rate is also recognised in these injuries together with significant adversities for these patients. Therefore, a well-organised multidirectional approach to this hazardous medical entity is essential in order to improve the outcomes in this cohort of elderly patients [10].

Despite the fact that significant evolution has been achieved in the surgical approaches and patient care lately, there is obscure evidence whether mortality rates are improving [9]. The results in the literature from studies generated from the existing hip fracture registries are conflicting. For instance, in a study by Daugaard et al. from 
2003 to 2010 , the 30 -day mortality rate was reported to be $10 \%$ in 38,020 patients, whereas in another study by Caretta et al. from 2004 to 2007, the 30-day mortality rate was $3.5 \%$ in 1320 patients $[11,12]$. With the implementation of specific hip fracture pathways to enhance the care of these frail elderly patients, one would argue that improvements in the morbidity and mortality rates should prevail.

The aim of this study therefore is to investigate whether the overall initiatives introduced during the past few years to improve the care of elderly patients with hip fractures have made an impact on the early (30 day) mortality rate.

\section{Patients and methods}

A literature review of relevant publications was carried out for the past 15 years. We used such 'mesh words' as early mortality rate, hip fractures, elderly, delay to surgery, 30-day mortality, early complications. Inclusion criteria were articles in English language, original papers published in peer-review journals that contained data about 30-day mortality after traumatic hip fractures in the elderly. Exclusion criteria were case reports, editorials, review articles and articles of hip fractures in young adults or children, articles about stress fractures of the proximal femur, as well as articles in other languages than in English. Moreover, we analysed the data of our hospital hip fracture database of elderly patients treated in our tertiary referral centre during the past 3 years.

It is of note that multiple variables are described in the literature to play an indispensable role in the 30-day mortality rates in different studies, although these variables are not encountered in every single study. Two major categories can be identified in these variables: variables that cannot be altered and referred to fixed patient characteristics and variables that can be changed and controlled [13]. Age, fracture type, gender, pre-fracture condition and mobility, date of admission (weekend or week days), mechanism of injury, accommodation and the Society of Anaesthesiologists score (ASA) are some of the parameters that cannot be altered, whereas grade of responsible surgeon and anaesthetist, time from the injury to operation, time from admission to operation and type of anaesthesia belong to the changing variables $[11,14,15]$.

\section{Results}

Initially 54 manuscripts were found to be relevant to this subject. However, following the application of the inclusion and exclusion criteria 15 manuscripts [9, 1113, 16-26] and data from 11 annual reports of the
Table 1 Data collected from international studies providing information about 30-day mortality rate after hip fracture

\begin{tabular}{llrl}
\hline Authors & Year & $\begin{array}{l}\text { No of } \\
\text { patients }\end{array}$ & $\begin{array}{l}\text { Early mortality } \\
\text { rate (\%) }\end{array}$ \\
\hline Charletton et al. & $2006-2013$ & 4426 & $6.5-12.1$ \\
Inacio et al. & $2009-2011$ & 12,562 & 6.2 \\
Tarrant et al. & 2011 & 437 & 8.4 \\
Khan et al. & $2009-2010$ & 467 & 7.5 \\
Lau et al. & $2009-2010$ & 759 & 2.5 \\
Daugaard et al. & $2003-2010$ & 38,020 & 10 \\
Choi et al. & $2002-2009$ & 874 & 1.4 \\
Miller et al. & $2006-2008$ & 338,092 & 8.1 \\
Castronuovo et al. & $2006-2007$ & 6896 & 7 \\
Caretta et al. & $2004-2007$ & 1320 & 3.5 \\
Nielsen et al. & $2005-2006$ & 6266 & 10.3 \\
Brauer et al. & $1986-2005$ & 786,717 & Female: 5.9-5.2 \\
& & & Male: 11.9-9.3 \\
Holt et al. & $1998-2004$ & 18,817 & 7 \\
Moran et al. & $1999-2003$ & 2660 & 9 \\
Gini et al. (Lazio) & $1999-2003$ & 32,019 & 5 \\
Gini et al. (Tuscany) & $1999-2003$ & 30,406 & 2.8 \\
\hline
\end{tabular}

National Hip Fracture Database (NHFD) formed the basis of this review.

From the data obtained, it appears that conflicting reports are recognised in many cases. For instance, Choi et al. found a very low (1.4\%) 30-day mortality rate, whereas Daugaard et al. noted an early mortality rate of $10 \%[11,21]$. Nonetheless, the majority of the most recent studies report that lately with the implementation of new guidelines and treatment protocols, a trend of a continuous decrease in the 30-day mortality rate of patients with hip fractures has been observed (Table 1). In agreement with this observation are the findings from the UK (based on the NHFD) (the largest hip fracture database in the world) [6]. A continuous steady reduction in the 30-day mortality rate has been shown as a result of the new multidisciplinary approach provided to the patients, the fastest time to surgery, the input in the medical treatment of the patients by the orthogeriatricians and the implementation of NICE guidelines for hip fractures (Table 2). Data obtained from our institution with regard to the 30-day mortality rate after hip fractures also demonstrated this trend (Table 3).

\section{Discussion}

Patients suffering from a hip fracture have a significantly higher early- and long-term mortality rate after the initial injury compared with healthy individuals of the same age with no fracture [19]. Currently, no consensus has been 
Table 2 Data collected from the National Hip Fracture Database (NHFD) in regard to 30-day mortality after hip fracture

\begin{tabular}{llll}
\hline Author & Year & No of patients & $\begin{array}{l}\text { 30-day mortality } \\
\text { rate }(\%)\end{array}$ \\
\hline NHFD & 2013 & 64,838 & 8.02 \\
NHFD & 2012 & 61,508 & 8.2 \\
NHFD & 2011 & 53,879 & 8.5 \\
NHFD & 2010 & 54,129 & 8.8 \\
NHFD & 2009 & 53,427 & 9.2 \\
NHFD & 2008 & 52,600 & 10.4 \\
NHFD & 2007 & 52,435 & 10.9 \\
NHFD & 2006 & 50,732 & 11.3 \\
NHFD & 2005 & 51,408 & 11.4 \\
NHFD & 2004 & 50,995 & 11.0 \\
NHFD & 2003 & 51,985 & 11.5 \\
\hline
\end{tabular}

As noted there is a continuous decrease in the 30-day mortality rate

reached with regard to the factors responsible for this difference in the mortality rate. This lack of consensus exists also in the established protocols for optimisation of patient care, the definition of early and late surgery, the implementation of rehabilitation protocols, the length of hospital stay, thus making it extremely difficult to compare data from registries with different diagnostic and treatment pathways. In a study of 18,817 patients by Holt et al. [13] in the Scottish Hip Fracture Audit Database, male sex, increased age and preoperative comorbidities (according to ASA) were all connected with a higher death risk. In the same study variables that can be changed in a patient suffering a hip fracture such as time to theatre and grade of surgeon and anaesthesiologist did not seem to affect the mortality rate in a significant way, whereas the period from the injury to the operation (and not the time from the hospital admission to the surgery) had a significant effect on 30-day mortality [13]. In an additional study from Holt et al. [27] 2 years later including 4284 patients the conclusion that patients with severe medical comorbidities were more likely to be operated late and faced an increased 30-day mortality rate was reported.

Noteworthy, White et al. [3] highlighted that despite medical advancements and the implementation of new fasttrack protocols, a rise in the 30-day mortality rate after surgical treatment of hip fractures should be expected from 8.3 to $8.9-9.3 \%$, adding $2200-7000$ deaths every year in the near future in the UK. The authors justified their projection on the following parameters: increased age of the population in the near future and increased age-related comorbidities [3].

In a recent pilot study from the Hip Fracture Accelerated Surgical Treatment and Care Track (HIP ATTACK) investigators, patients who have been treated in the first $6 \mathrm{~h}$ after the injury (accelerated care group) had significantly lower 30-day mortality rate compared to the standard care group ( 3 and $13 \%$, respectively) (the mean time to surgery for the accelerated group was $6 \mathrm{~h}$ compared to the $24,2 \mathrm{~h}$ for the standard group) [28]. This was attributed to a very aggressive early medical optimisation and early operative treatment of the hip fracture [28].

With regard to the impact on mortality of the date of admission parameter, reports from the literature are contradictive. In a study by Clarke et al. in 2010, no significant difference was found between admission on weekdays and weekends, whereas Schilling et al. in 2010 reported an increased mortality rate for weekend admissions in a total of 166,920 patients $20 \%$ of whom suffered from hip fractures $[29,30]$.

Guidelines established in New Zealand and Scottish Health Care System recommend early surgery (within the first $24 \mathrm{~h}$ of the injury) for the reduction of postoperative complications and mortality [31, 32]. However, the reports in the literature seem to be controversial. On the one hand, there is a meta-analysis performed by Shiga et al. that reported a $41 \%$ increase in the 30-day mortality rate and a $32 \%$ increase in the 1-year mortality rate after delayed surgery for a patient with a hip fracture, but on the other hand, there are several studies that did not demonstrate a connection between the treatment time and postoperative complications and mortality [26, 33, 34]. With regard to the development of complications, the respiratory system, the cardiovascular system, the urogenital and the central nervous system are mostly involved, with the operation to have a significant stress to all of the systems [19].

In a systematic review of 52 published studies that included 291,413 patients in total, a suggestion was made that hip surgery should take place within the first $48 \mathrm{~h}$ after injury in order to expect a reduction in the rate of complications and overall mortality [19]. The risk of 30-day mortality in a Danish study of 38,020 patients with hip fracture increased with an OR of 1.3 per 24-h surgical delay [11]. Similarly, in a recent study by Nyholm et al. [35] even a delay in the operation for more than $12 \mathrm{~h}$
Table 3 Data regarding the 30-day mortality rate of patients treated in the Leeds General Infirmary (LGI)

\begin{tabular}{lllll}
\hline LGI & Year & Hip admissions & Deaths at 30 days & 30-day mortality rate (\%) \\
\hline NHFD & 2015 (till November) & 638 & 44 & 6.7 \\
NHFD & 2014 & 715 & 50 & 6.9 \\
NHFD & 2013 & 678 & 74 & 10 \\
\hline
\end{tabular}


increased the 30-day mortality rate compared to the patients operated within $12 \mathrm{~h}$ from the injury. It is of interest that another study from Sweden showed that longer length of hospital stay seems to be beneficial for patients with regard to early mortality rates [36].

For the type of anaesthesia and the possible connection to early mortality rate, a meta-analysis of 22 trials performed by Parker et al. [37] including 2567 patients could not report a safe conclusion due to insufficient evidence available from the studies.

Considerable debate has been noted also in the grade of the orthopaedic surgeon and the anaesthetist treating patients with hip fractures. There have been data suggesting that patients treated by junior anaesthetists experience lower 30-day mortality rates than other patients treated by senior staff $[13,27]$. However, this finding could be connected with the fact that senior staff is more likely to handle patients with significant comorbidities [13]. In the same study, when the results in patient care mix were calculated with the multivariate model, no significant difference was noted in the 30-day mortality regarding the staff grade [13].

It is of interest that there is no clear consensus in the literature with regard to the early surgery definition. A hospital statistics analysis of 129,522 patients performed by Bottle et al. [38] showed a higher risk of 30-day mortality in patients operated after $24 \mathrm{~h}$. In another study by McGuire et al. [39] in 18,209 patients with hip fracture increased early mortality rate was reported for patients receiving delayed surgery (after $48 \mathrm{~h}$ from the initial injury). On the other hand, there is evidence to support that early surgery does not play an important role in short-term mortality rates. For instance, Holt et al. [13, 27] reported that no clear effect of the time of operation and 30-day mortality could be found, with the time to surgery to be representing a chance association. Therefore, new studies are essential in order to be able to specify whether the over 48-h operation delay is an independent risk factor for increased mortality and also the relationship between time to operation and early mortality in the case mix [40].

Preoperative factors that can influence postoperative complications and mortality such as increased age, poor pre-injury functional level, multiple medical comorbidities and male gender seem to play a significant role in increasing early mortality rates [13, 20, 41, 42]. For patients with hip fractures that are treated non-operatively, the reported 30-day mortality rate is significantly higher $(34 \%)$ than the 30-day mortality rate of the patients treated operatively [43].

Recent changes in the UK Medical Care in this elderly group of patients led to the improvement of the outcomes of patients with hip fractures. These changes include the national introduction of NHFD, the British Orthopaedic Associations guidelines for fragility fractures and the best practise tariff for hip fractures $[5,6]$. The latter includes a geriatrician review for the patient within the first $72 \mathrm{~h}$, operative treatment of the hip fracture within the first $36 \mathrm{~h}$, multispecialty protocol approved by geriatricians, orthopaedics and anaesthetists, evaluation of underlying bone fragility and advanced rehabilitation programmes.

NHFD represents a firmly organised and clinically led quality improvement initiative with main purpose to document different aspects in patients with hip fractures and implement well-established techniques and protocols for the improvement of the outcomes. In the UK, there are 182 hospitals that subscribe in the NHFD and provide monthly data, making the NHFD the largest hip fracture database in the world [6]. Hospital facilities and staff are in continuous evaluation for the implementation of the protocols provided, and audits are performed regularly for multidirectional evaluation of the standard of care provided.

For a better patient assessment and mortality predictor the Nottingham Hip Fracture Score seems to provide important help in regard to 30-day mortality. Variables such as increased age, the presence of 2 or more comorbidities, male gender, low values of haemoglobin and a mental test $<6$ might be independent predictors of mortality [44]. In another study by Lau et al. [41], it has been reported that the total Charlson comorbidity score (TCCS) correlates with early and late mortality. TCCS is important demonstrating the general condition of the patient on the day of admission after sustaining a hip fracture. Therefore, in patients with multiple comorbidities and high TCCS increased short-term mortality rate is expected. The TCCS is proposed as a reliable predictor of 30-day and 1-year mortality in geriatric patients sustaining a hip fracture [41].

In conclusion, it appears that following the implementation of fast-track care pathways, input from orthogeriatricians, quick patient medical optimisation, combined input by orthopaedic surgeons, anaesthetists, orthogeriatrician and early surgery (earlier than $36 \mathrm{~h}$ from the injury) and advanced rehabilitation protocols, there has been a reduction in the 30-day mortality rate of elderly patients undergoing surgery for hip fractures. A number of parameters have contributed to this downward trend noted. Whether we have reached a plateau or whether a steady ongoing reduction in mortality rates will continue is yet to be seen.

\section{Compliance with ethical standards}

Ethical approval As this study was based on review of the literature, no ethical approval was needed.

Conflict of interest Dionysios Giannoulis and Giorgio M Calori declare that they have no conflict of interest. Peter V Giannoudis has 
received research grant from Zimmer Biomet and Depuy- Synthes. He is a member of the Editorial Board of Injury (Editor in Chief). He reports no other conflict of interest.

Open Access This article is distributed under the terms of the Creative Commons Attribution 4.0 International License (http://crea tivecommons.org/licenses/by/4.0/), which permits unrestricted use, distribution, and reproduction in any medium, provided you give appropriate credit to the original author(s) and the source, provide a link to the Creative Commons license, and indicate if changes were made.

\section{References}

1. Tosounidis TH, Castillo R, Kanakaris NK, Giannoudis PV (2015) Common complications in hip fracture surgery: tips/tricks and solutions to avoid them. Injury 46(Suppl 5):S3-S11

2. Tosounidis TH, Sheikh H, Stone MH, Giannoudis PV (2015) Pain relief management following proximal femoral fractures: options, issues and controversies. Injury 46(Suppl 5):S52-S58

3. White SM, Griffiths R (2011) Projected incidence of proximal femoral fracture in England: a report from the NHS hip fracture anaesthesia network (HIPFAN). Injury 42(11):1230-1233. doi:10.1016/j.injury.2010.11.010

4. Edwards TC (2009) Bureau reports: world's older population projected to triple by 2050. 2009(1). CB09-97. http://www.cen sus.gov/Press-Release/www/releases/archives/international_popu lation/013882.html

5. British Orthopaedic Association Standards for Trauma (BOAST 1) Guideline (2008). Available online at http://www.boa.ac.uk/LIB/ LIBPUB/Documents/BOAST1-HipFractureintheOlderPerson,Version1-2008.pdf. Last Accessed 20 Dec 2012

6. No Authors Listed. The National Hip Fracture Database National Reports (2014, 2013, 2012, 2011, 2010, 2009). Available online at http://www.nhfd.co.uk/003/hipfractureR.nsf/NHFDNationalRe port2. Accessed Sep 2014

7. Hommel A, Ulander K, Bjorkelund KB, Norrman PO, Wingstrand H, Thorngren KG (2008) Influence of optimised treatment of people with hip fracture on time to operation, length of hospital stay, reoperations and mortality within 1 year. Injury 39(10):1164-1174

8. National Institute for Health and Care Excellence (NICE). Quality Standards for Hip Fracture 2015. http://www.nice.org.uk. Accessed Dec 2015

9. Brauer C, Perraillon M, Cutler D, Rosen A (2009) Incidence and mortality of hip fractures in the United States. JAMA 302(14):1573-1579. doi:10.1001/jama.2009.1462

10. Schnell S, Friedman S, Mendelson D, Bingham K, Kates S (2010) The 1-year mortality of patients treated in a hip fracture program for elders. Geriatr Orthop Surg Rehabil 1(1):6-14. doi:10.1177/ 2151458510378105

11. Daugaard CL, Jørgensen HL, Riis T, Lauritzen JB, Duus BR, Van Der Mark S (2012) Is mortality after hip fracture associated with surgical delay or admission during weekends and public holidays? A retrospective study of 38, 020 patients. Acta Orthopaedica 83(6):609-613

12. Carretta E, Bochicchio V, Rucci P, Fabbri G, Laus M, Fantini MP (2011) Hip fracture: effectiveness of early surgery to prevent 30-day mortality. Int Orthop 35(3):419-424. doi:10.1007/s00264010-1004-x (Epub 2010 Apr 8)

13. Holt G, Smith R, Duncan K, Finlayson DF, Gregori A (2008) Early mortality after surgical fixation of hip fractures in the elderly: an analysis of data from the Scottish hip fracture audit. J Bone Joint Surg Br 90(10):1357-1363. doi:10.1302/0301-620X. 90B10.21328

14. Hu F, Jiang C, Shen J, Tang P, Wang Y (2012) Preoperative predictors for mortality following hip fracture surgery: a systematic review and meta-analysis. Injury 43(6):676-685

15. Cheng T, Zhang GY, Liu T, Zhang XL (2012) A meta-analysis of percutaneous compression plate versus sliding hip screw for the management of intertrochanteric fractures of the hip. J Trauma Acute Care Surg 72:1435-1443

16. Chatterton BD, Moores TS, Ahmad S, Cattell A, Roberts PJ (2015) Cause of death and factors associated with early in-hospital mortality after hip fracture. Bone Joint J. 97(B(2)):246-251. doi:10.1302/0301-620X.97B2.35248

17. Inacio MCS, Weiss MJ, Miric A, Hunt JJ, Zohman GL, Paxton EW (2015) A community-based hip fracture registry: population, methods, and outcomes. Perm J 19(3):29-36. doi:10.7812/TPP/ 14-231

18. Tarrant SM, Hardy BM, Byth PL, Brown TL, Attia J, Balogh ZJ (2014) Preventable mortality in geriatric hip fracture inpatients. Bone Joint J 96(B(9)):1178-1184. doi:10.1302/0301-620X.96B9. 32814 (Published online 2014 September 1)

19. Khan SK, Kalra S, Khanna A, Thiruvengada MM, Parker MJ (2009) Timing of surgery for hip fractures: a systematic review of 52 published studies involving 291,413 patients. Injury 40(7):692-697. doi:10.1016/j.injury.2009.01.010

20. Lau TW, Fang C, Leung F (2013) The effectiveness of a geriatric hip fracture clinical pathway in reducing hospital and rehabilitation length of stay and improving short-term mortality rates. Geriatr Orthop Surg Rehabil 4:3-9. doi:10.1177/2151458513484759

21. Choi HJ, Kim E, Shin YJ, Choi BY, Kim YH, Lim TH (2014) The timing of surgery and mortality in elderly hip fractures: a retrospective, multicenteric cohort study. Indian J Orthop 48(6):599-604. doi:10.4103/0019-5413.144232

22. Miller JB, Cai X, Cram P (2012) Mortality rates are similar after hip fractures for rural and urban patients. Clin Orthop Relat Res 470(6):1763-1770. doi:10.1007/s11999-011-2140-3 (Published online 2011 October 21)

23. Castronuovo E, Pezzotti P, Franzo A, Di Lallo D, Guasticchi G (2011) Early and late mortality in elderly patients after hip fracture: a cohort study using administrative health databases in the Lazio region, Italy. BMC Geriatr 11:37. doi:10.1186/14712318-11-37 (Published online 2011 August 5)

24. Nielsen KA, Jensen NC, Jensen CM, Thomsen M, Pedersen L, Johnsen SP, Ingeman A, Bartels PD, Thomse RW (2009) Quality of care and 30 day mortality among patients with hip fractures: a nationwide cohort study. BMC Health Serv Res 9: 186, Published online 2009 Oct 12. doi: 10.1186/1472-6963-9-186PMCID: PMC2768699

25. Moran CG, Wenn RT, Sikand M, Taylor AM (2005) Early mortality after hip fracture: is delay before surgery important? J Bone Joint Surg Am 87(3):483-489. doi:10.2106/JBJS.D.01796

26. Gini R, Capon A, Roti L, Mastromattei A, Buiatti E (2007) Femur fractures among elderly in Lazio and Tuscany regions from 1999 to 2003. Epidemiol Prev 31(4):197-203

27. Holt G, Smith R, Duncan K, McKeown DW (2010) Does delay to theatre for medical reasons affect the peri-operative mortality in patients with a fracture of the hip? J Bone Joint Surg $\mathrm{Br}$ 92:835-841. doi:10.1302/0301-620X.92B6.24463

28. Buse GL, Bhandari M, Sancheti P, Rocha S, Winemaker M, Adili A, de Beer J, Tiboni M, Neary JD, Dunlop V, Gauthier L, Patel A, Robinson A, Rodseth RN, Kolesar R, Farrell J, Crowther M, Tandon V, Magloire P, Dokainish H, Joseph P, Tomlinson CW, Salehian O, Hastings D, Hunt DL, Van Spall H, Cosman TL, Simpson DL, Cowan D, Guyatt G, Alvarado K, Evans WK, Mizera R, Eikelboom J, Cook D, Loeb M, Johnstone J, Kearon C, 
Sessler DI, VanHelder T, Rao-Melacini P, Worster A, Patil A, McLean R, Macdonald AM, Badzioch R, Devereaux PJ (2014) Accelerated care versus standard care among patients with hip fracture: the HIP ATTACK pilot trial, Hip Fracture Accelerated Surgical Treatment and Care Track (HIP ATTACK) Investigators. CMAJ 186(1):E52-E60. doi:10.1503/cmaj.130901 (Epub 2013 Nov 18)

29. Clarke MS, Wills RA, Bowman RV, Zimmerman PV, Fong KM, Coory MD, Yang IA (2010) Exploratory study of the 'weekend effect' for acute medical admissions to public hospitals in Queensland, Australia. Intern Med J. 40(11):777-783. doi:10. 1111/j.1445-5994.2009.02067.x

30. Schilling PL, DA Campbell JR, Englesbe MJ, Davis MM (2010) A comparison of in-hospital mortality risk conferred by high hospital occupancy, differences in nurse staffing levels, weekend admission, and seasonal influenza. Med Care 48(3):224-232. doi:10.1097/MLR.0b013e3181c162c0

31. New Zealand Guidelines Group (2003) Acute management and immediate rehabilitation after hip fracture amongst people aged 65 years and over. Wellington, NZ, guidelines/0007/Hip_Fracture_Management_Fulltext.pdf. Accessed 11 Mar 2010

32. Scottish Intercollegiate Guideline Network (2009) Management of hip fracture in older people. A national clinical guideline. http://www.sign.ac.uk/guidelines/fulltext/111/index.html. Accessed 21 Jul 2009

33. Franzo A, Francescutti C, Simon G (2005) Risk factors correlated with post-operative mortality for hip fracture surgery in the elderly: a population-based approach. Eur J Epidemiol 20(12):985-991

34. Shiga T, Wajima Z, Ohe Y (2008) Is operative delay associated with increased mortality of hip fracture patients? Systematic review, meta-analysis, and meta-regression. Can J Anaesth 55(3):146-154

35. Nyholm AM, Gromov K, Palm H, Brix M, Kallemose T, Troelsen A (2015) Danish fracture database collaborators, time to surgery is associated with thirty-day and ninety-day mortality after proximal femoral fracture: a retrospective observational study on prospectively collected data from the danish fracture database collaborators. J Bone Joint Surg Am 97(16):1333-1339. doi:10. 2106/JBJS.O.00029

36. Nordström P, Gustafson Y, Michaelsson K, Nordström A (2015) Length of hospital stay after hip fracture and short term risk of death after discharge: a total cohort study in Sweden. BMJ 350:h696. doi:10.1136/bmj.h696

37. Parker MJ, Handoll HH, Griffiths R (2004) Anaesthesia for hip fracture surgery in adults. Cochrane Database Syst Rev 4:CD000521. doi:10.1002/14651858.CD000521.pub2

38. Bottle A, Aylin P (2006) Mortality associated with delay in operation after hip fracture: observational study. BMJ 332(7547):947-951. doi:10.1136/bmj.38790.468519

39. McGuire KJ, Bernstein J, Polsky D, Silber JH (2004) The 2004 Marshall Urist award: delays until surgery after hip fracture increases mortality. Clin Orthop Relat Res 428:294-301. doi:10. 1097/01.blo.0000146743.28925.1c

40. Bryson GL (2008) Waiting for hip fracture repair-do outcomes and patients suffer? Can J Anaesth 55(3):135-139. doi:10.1007/ BF03016086

41. Lau TW, Fang C, Leung F (2015) Assessment of postoperative short-term and long-term mortality risk in Chinese geriatric patients for hip fracture using the Charlson comorbidity score. Hong Kong Med J. doi:10.12809/hkmj154451

42. Kannegaard PN, van der Mark S, Eiken P, Abrahamsen B (2010) Excess mortality in men compared with women following a hip fracture. National analysis of comedications, comorbidity and survival. Age Ageing 39:203-209

43. Gregory JJ, Kostakopoulou K, Cool P, Ford DJ (2010) One-year outcome for elderly patients with displaced intracapsular fractures of the femoral neck managed non-operatively. Injury 41(12):1273-1276

44. Rushton PR, Reed MR, Pratt RK (2015) Independent validation of the Nottingham hip fracture score and identification of regional variation in patient risk within England. Bone Joint $\mathrm{J}$ 97(B(1)):100-103. doi:10.1302/0301-620X.97B1.34670 